\title{
Molecular Characteristic and Virulence Gene Profiles of Community-Associated Methicillin-Resistant Staphylococcus aureus Isolates from Pediatric Patients in Shanghai, China
}

\author{
Xing Wang ${ }^{1 *}$, Xia $\mathrm{Li}^{2}$, Wei Liu ${ }^{1}$, Weichun Huang ${ }^{1}$, Qihua $\mathrm{Fu}{ }^{1}$ and $\mathrm{Min} \mathrm{Li}^{3 *}$ \\ ${ }^{1}$ Department of Laboratory Medicine, Shanghai Children's Medical Center, Shanghai Jiaotong University School of Medicine, \\ Shanghai, China, ${ }^{2}$ Department of Medical Microbiology and Immunology, University of California, Davis, Davis, CA, USA, \\ ${ }^{3}$ Department of Laboratory Medicine, Renji Hospital, Shanghai Jiaotong University School of Medicine, Shanghai, China
}

OPEN ACCESS

Edited by:

Johnan A. R. Kaleeba

Uniformed Services University of the

Health Sciences, USA

Reviewed by:

Jesús Santos,

University of León, Spain

Pallab Ghosh

Harvard Medical School, USA

${ }^{*}$ Correspondence:

Xing Wang

wx_5166@163.com

$\mathrm{Min} \mathrm{Li}$

ruth_limin@126.com

Specialty section:

This article was submitted to

Infectious Diseases,

a section of the journal

Frontiers in Microbiology

Received: 10 August 2016

Accepted: 28 October 2016

Published: 15 November 2016

Citation:

Wang X, Li X, Liu W, Huang W, Fu Q and Li M (2016) Molecular

Characteristic and Virulence Gene

Profiles of Community-Associated

Methicillin-Resistant Staphylococcus

aureus Isolates from Pediatric Patients

in Shanghai, China

Front. Microbiol. 7:1818.

doi: 10.3389/fmicb.2016.01818
Staphylococcus aureus is a globally important human pathogen, especially among children and immunocompromised patients. The emergence and spread of community-associated methicillin-resistant S. aureus (CA-MRSA) has become a serious public health problem worldwide. The aim of this study was to investigate the prevalence, molecular characteristics and virulence profiles of CA-MRSA infections from pediatric patients in a university hospital in Shanghai, China. A total of 80 CA-MRSA isolates were collected from July 2012 to December 2013 in Shanghai Children's Medical Center and analyzed by multilocus sequence typing, staphylococcus chromosomal cassette mec (SCCmec) typing, and spa typing. The detection of Panton-Valentine Leukocidin (pvl), superantigenic and exfoliative toxins, and adhesin genes was also performed. Overall, 16 distinct sequence types (STs) were identified among the 80 isolates. Among them, ST59 was found to be the most prevalent, followed by ST398 $(11.3 \%, 9 / 80)$ and ST88 (8.8\%, 7/80). SCCmec types IV and $V$ were observed, at 60 and $40 \%$, respectively. Thirty spa types were identified, spa $t 437$ (23.8\%) was the most predominant type. All 80 isolates exhibited carriage of at least four virulence genes. Thirty-four $(42.5 \%, 34 / 80)$ isolates harbored $\geq 10$ tested virulence genes. Adhesion genes were present in most of the MRSA isolates, including the following: icaA (100\%), clfA (100\%), sdrC (95\%), and sdrE (63.8\%). The prevalence of pvl gene was 20\%, and multidrug resistance was observed in $36 \%$ of all strains. In addition, ST59-MRSA-IV with $t 437$ accounted for $21.3 \%$ of occurrences, making it the most prevalent clone. Isolates that were carriers of toxin genes, and hla (100\%) and hlg (87.5\%) were the most frequent. In conclusion, simultaneous carriage of multiple virulence genes and genetically considerable diversity were very common among CA-MRSA from pediatric patients in Shanghai. ST59-MRSA-IV with $t 437$ was still the most predominant type. The combination of virulence gene profiles and antibiotic resistance may help ST59 to be successfully spread among children.

Keywords: community-associated methicillin-resistant staphylococcus aureus, MLST-genotyping, SCCmec typing, spa typing, virulence factors 


\section{INTRODUCTION}

Staphylococcus aureus is one of the most prevalent human pathogens, causing a broad variety of diseases ranging from mild skin and soft-tissue infections to severe systemic infections such as sepsis and necrotizing pneumonia (Lowy, 1998; Deresinski, 2005). Since methicillin-resistant S. aureus (MRSA) was first reported in the United Kingdom in 1961 (Monecke et al., 2011), it has become a particular public threat to human health. During the last 55 years, various hospital-associated MRSA (HA-MRSA) clones have disseminated worldwide, including Europe, United States, North Africa, the Middle East, and East Asia. Since the 1990s, community-associated MRSA (CA-MRSA) emerged as a serious health problem worldwide (David and Daum, 2010; DeLeo et al., 2010), first in communities and later in healthcare facilities. Compared to HA-MRSA strains, most CAMRSA isolates that harbor SCCmec types IV or V, do not have multi-antibiotic resistance (except to $\beta$ lactams), and many possess different exotoxin gene profiles (e.g., PVL genes; Dinges et al., 2000). However, another MRSA clone from animals (LAMRSA) has emerged in humans exposed to livestock since 2005. Livestock-associated MRSA ST398, isolated from pigs and pig farmers has been reported in European countries and North America. For LA-MRSA, ST398 is the overwhelmingly dominant lineage in Europe, whereas ST9 predominates in most Asian countries (Fluit, 2012; Chuang and Huang, 2015).

Currently, more than 20 genetic lineages have been reported to be associated with CA-MRSA worldwide (Witte, 2009; Mediavilla et al., 2012). The top five major lineages include ST1-IV (WA1, USA400), ST8-IV (USA300), ST30-IV (South West Pacific clone), ST59-V (Taiwan clone), and ST80-IV (European clone). However, CA-MRSA strains were found to be continent-specific. For example, ST1 and ST8 clones are mostly found in the United States and Canada, whereas ST80 clones are mainly found in Europe. In China and several other Asian countries, ST59IV and ST59-V are the most common CA-MRSA strains. The prevalence of CA-MRSA varies greatly and the occurrence rate of CA-MRSA infections ranges from $<5$ to $>35 \%$ in different Asian countries (Chen and Huang, 2014). Prevalence is higher in children than in adults. Because of low immunity in children, once infected with CA-MRSA, the consequences are often very serious (Machuca et al., 2013). Although, CA-MRSA infection and transmission represent a major public health problem, there is limited information reported on CA-MRSA monitoring among children in China. The aim of this study was to investigate the prevalence, molecular characteristics and virulence profiles of CA-MRSA infections from pediatric patients in China and to determine whether the epidemiology could be affected by time.

\section{MATERIALS AND METHODS}

\section{Bacterial Isolates}

From July 2012 to December 2013, a total of 80 sequential CAMRSA isolates, which represent all the non-duplicate strains isolated during the study period, were collected from pediatric patients with local or systemic infections in a university hospital in Shanghai, China (Shanghai Children's Medical Center, affiliated with Shanghai Jiao Tong University; CLSI, 2012). Shanghai Children's Medical Center is one of the largest pediatric hospitals in China with 800 beds and $\sim 3000$ hospital admissions per day. MRSA isolates were confirmed by classic microbiological methods: Gram stain and catalase and coagulase activity on rabbit plasma. They were further identified by biochemical characterization using the Api-Staph test (bioMérieux, Lyon, France).

CA-MRSA was defined as an MRSA isolate that was obtained either from an outpatient or an inpatient within $48 \mathrm{~h}$ of hospitalization, and without the patient having medical history of MRSA infection or colonization, admission to a healthcare facility, dialysis, surgery or insertion of indwelling devices in the past year.

These isolates were recovered from several clinical sources, including the respiratory tract (sputum, pharynx swabs, and bronchial alveolar lavage fluid), skin and soft tissue (cutaneous abscess and wound secretion), cerebrospinal fluid, blood and urine (Klevens et al., 2007). All strains were stored at $-70^{\circ} \mathrm{C}$ and grown overnight on sheep blood agar plates at $37^{\circ} \mathrm{C}$.

The Ethics Committee of Shanghai Children's Medical Center exempted this study from review because the present study focused on bacteria.

\section{Antimicrobial Susceptibility Testing}

The antibiotic susceptibility of all isolates in this study was performed using the bioMe'rieux VITEK2 system following manufacturer's instructions. Results were interpreted in accordance with Clinical and Laboratory Standards Institute (CLSI) guidelines (CLSI, 2012). The following 17 drugs were tested: cefazolin (CFZ), linezolid (LZD), ciprofloxacin (CIP), clindamycin (DA), erythromycin (E), trimethoprimsulfamethoxazole (SXT), moxifloxacin (MOF), nitrofurantoin $(\mathrm{FD})$, vancomycin $(\mathrm{V})$, tetracycline (TET), penicillin (P), rifampicin (RF), levofloxacin (LVX), ampicillin (AMP), gentamicin (GM), quinupristin/dalfopristin (Q/D), and tigecycline (TGC). S. aureus ATCC 29213 was used as a quality control.

\section{MLST Analysis}

Isolates were screened according to the protocol described (Enright and Spratt, 1999) on the S. aureus MLST website (http://saureus.mlst.net) to detect the following seven housekeeping genes (Aanensen and Spratt, 2005): carbamate kinase $(\operatorname{arcC})$, shikimate dehydrogenase $(\operatorname{aroE})$, glycerol kinase $(g l p)$, guanylate kinase $(g m k)$, phosphate acetyltransferase (pta), triosephosphate isomerase (tpi), and acetyl coenzyme A acetyltransferase (yqiL). PCR amplicons of seven $S$. aureus housekeeping genes were obtained from chromosomal DNA. The sequences of the PCR products were compared with the existing alleles available from the MLST website, and the allelic number (sequence type, ST) was determined for each sequence. Clustering of related STs, which were defined as clonal complexes (CCs), was determined using eBURST (based on related STs). 


\section{SCCmec Typing}

The MRSA isolates were subjected to SCCmec typing as described by Kondo et al. (2007), which was based on a set of multiplex PCRs (M-PCRs) with 14 primers. SCCmec types I$\mathrm{V}$ were assigned according to the combination of the cassette chromosome recombinase ( $c c r$ ) type and mec class. MRSA isolates that could not be assigned to any expected type were defined as nontypable (NT).

\section{Spa Typing}

In $S$. aureus, the polymorphic X region of staphylococcal protein A (spa) gene consists of a variable number of 24 bp repeat units (Shopsin et al., 1999) that allow isolates to be distinguished from one another. The spa typing was based on variations of the repeat units. Amplification and sequencing of the $\mathrm{X}$ region were performed as described previously by Koreen et al. (2004). The spa typing was assigned by submitting the data to the $S$. aureus spa type database (http://spaserver.ridom.de).

\section{Virulence Gene Profiles}

All MRSA isolates were screened for the following 22 staphylococcal virulence genes: the staphylococcal enterotoxin genes (sea, seb, sec, sed, see, seg, seh, sei, seq, sek), the toxic shock syndrome toxin $(t s s t)$, the arginine catabolic mobile gene $(\operatorname{arc} A)$, the exfoliative toxin genes (eta, etb), the PVL genes (lukF/S-PV; Lina et al., 1999), the hemolysin gene ( $h l a, h l b, h l g)$, and the adhesin genes ( $c l f A, i c a A, s d r C$, and $s d r E$ ) as previously described (Arvidson and Tegmark, 2001; Peacock et al., 2002; Bubeck Wardenburg et al., 2007).

\section{Statistical Analysis}

Statistical analyses were performed using Stata software (version 10.1/SE, Stata Corp., College Station, TX, USA). We used the $\chi^{2}$ and Fisher's exact tests, as appropriate for analysis of categorical data. Statistical significance was set at $P \leq 0.05$.

\section{RESULTS}

\section{Clinical Features}

Eighty MRSA isolates were obtained from patients 10 days to 11 years old with local or systemic infection and all the isolates were positive for the mecA genes. Among them, 52 patients (65\%) were male. $61.3 \%(49 / 80)$ of the children were $<1$ year old and 18 were neonates $(22.5 \%)$. From the clinical medical records, respiratory infection was the most frequently determined infection type caused by CA-MRSA; $82.5 \%$ (66/80) of the isolates were from the respiratory tract, and $5 \%(4 / 80)$ of the isolates were associated with bloodstream infection (BSI).

\section{MLST, SCCmec, and Spa Typing}

The evolutionary and genetic diversity of MRSA isolates within individual patients was analyzed by MLST (Table 1). There were 16 distinct STs identified within the 80 isolates, among which the most frequently represented was ST59 $(45.0 \%, 36 / 80)$. It accounted for nearly one half of all MRSA isolates, followed by ST398 (11.3\%, 9/80), ST88 (8.8\%, 7/80), ST5 (7.5\%, 6/80), ST630 $(7.5 \%, 6 / 80)$, ST7 $(3.8 \%, 3 / 80)$, ST338 $(2.5 \%, 2 / 80)$, ST45 $(2.5 \%$,
2/80), ST9 (2.5\%, 2/80). Among the remaining 7 STs (ST30, ST72, ST6, ST20, ST950, ST1507, ST121), each ST just had one isolate. This methodology revealed that the strains clustered by eBURST into 9 CCs (CC59, CC398, CC88, CC5, CC8, CC7, CC72, CC45, CC9) and 4 singletons (Figure 1), among which were CC59 with 38 isolates, CC398 with 9 isolates, CC88 with 7 isolates, CC5 with 7 isolates, CC8 with 6 isolates, CC7 with 3 isolates, CC72 with 2 isolates, CC45 with 2 isolates, and CC9 with 2 isolates. Sixteen isolates harboring $p v l$ were distributed among 7 different STs, including ST59 $(n=9)$ and ST338 $(n=2)$, as well as ST398, ST88, ST30, ST20, and ST121 (one isolate each).

By SCCmec typing, only two types (types IV and V) were found among 80 MRSA isolates. The most common was type IV, which was found in 48 isolates $(60 \%, 48 / 80)$, while type V was found in 32 isolates $(40 \%, 32 / 80)$.

The spa typing discriminated MRSA isolates into 30 spa types. Among them, spa $t 437$ was the most predominant type $(23.8 \%$, $19 / 80)$, followed by $t 441(8.8 \%, 7 / 80), t 034(7.5 \%, 6 / 80), t 4549$ (6.3\%, 5/80), $t 172(5 \%, 4 / 80), t 316$ (5\%, 4/80), and t2310 (5\%, $4 / 80)$. Each of the remaining spa types was represented in $<3$ isolates.

There was a strong association observed between specific ST and spa types. The ST59 genotype was associated primarily with spa t437 $(47.2 \%, 17 / 36)$ and spa t441 $(19.4 \%, 7 / 36)$, and less frequently with six types: $t 316, t 172, t 163, t 3523, t 8886, t 1751$. The ST398 genotype was associated mainly with spa t034 (6/9) and spa t011 (2/9).

\section{Antimicrobial Susceptibility Testing}

The antimicrobial resistance profiles of 80 MRSA isolates according to MLST are listed in Table 2. All the strains were resistant to cefazolin, penicillin, and ampicillin, but susceptible to vancomycin, linezolid, nitrofurantoin, quinupristin/dalfopristin, and tigecycline. The majority were resistant to clindamycin (80\%) and erythromycin (82.5\%), however, they were susceptible to most of the antibiotics tested. The resistance rates to other antibiotics tested were $8.8 \%$ to ciprofloxacin, $10 \%$ to trimethoprim-sulfamethoxazole, $3.8 \%$ to moxifloxacin, $32.5 \%$ to tetracycline, $2.5 \%$ to rifampicin, $5 \%$ to levofloxacin, and $3.8 \%$ to gentamicin. The resistance profiles of MRSA isolates differed by their STs. For the ST59 strains, the most prevalent antibiotic group contains clindamycin, erythromycin, and tetracycline, while ST9 strains were more resistant to more antibiotics including ciprofloxacin, clindamycin, erythromycin, trimethoprim-sulfamethoxazole and tetracycline.

\section{Virulence Gene Profiles}

The distribution of 19 putative virulence genes differed among the 80 MRSA strains according to MLST (Table 3). All virulence genes except eta, etb, and $\operatorname{arcA}$ genes were identified within multiple isolates, and all isolates exhibited carriage of at least 4 virulence genes. Thirty-four $(42.5 \%, 34 / 80)$ isolates harbored $\geq 10$ tested virulence genes, among which were 1 isolate with 13 genes, 3 isolates with 12 genes, 14 isolates with 11 genes, and 16 isolates with 10 genes.

Adhesion genes were present in most of the MRSA isolates; $100 \%$ carried the icaA and $c l f A$ genes, 95\% harbored $s d r C, 63.8 \%$ 


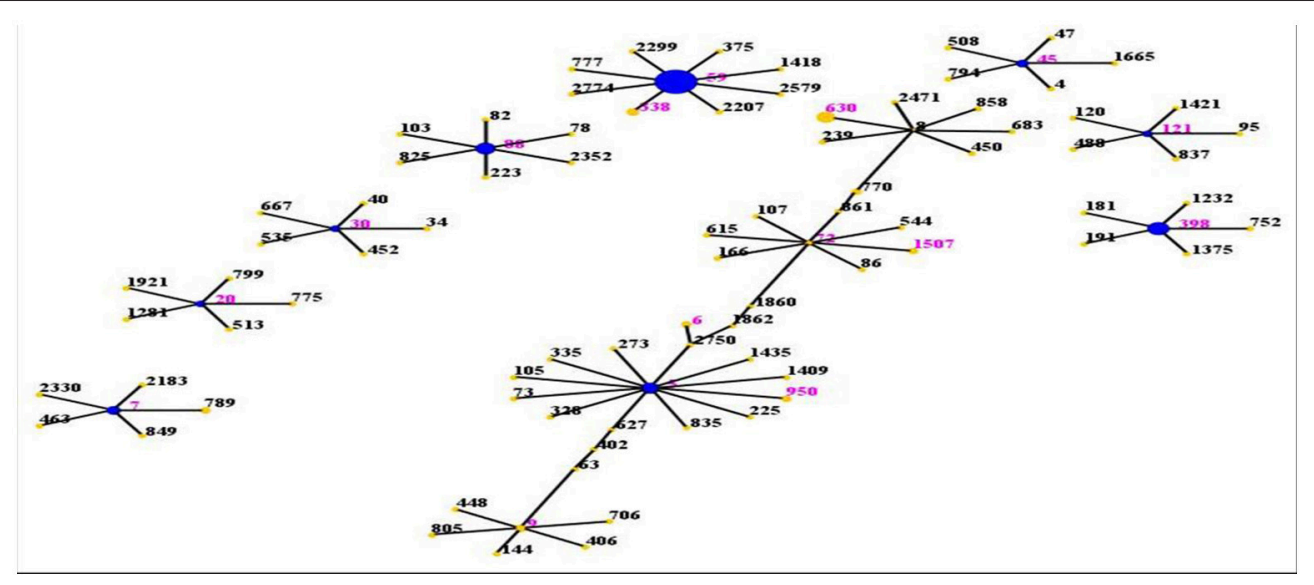

FIGURE 1 | Distribution of STs in the clonal complexes. The eBURST application of the MLST data from all of the isolates analyzed in this study. The purple numbers represent 16 STs which are found in 80 MRSA isolates. STs that are linked by a line belong to the same cluster. Circle sizes are proportional to the number of strains within the ST.

carried $s d r E$. The most prevalent toxin genes detected were hla (100\%), hlg (87.5\%), hlb (60\%), seb (46.3\%), sek (41.3\%), and seq $(41.3 \%)$. The $p v l$ gene was detected in 16 strains, which represented seven different STs, with ST59 being the most common.

\section{Molecular Characteristics of the Prevalent Clone ST59}

In this study, ST59 $(45.0 \%, 36 / 80)$ was found to be the most prevalent clone, which was associated primarily with spa $t 437$ $(44.7 \%, 17 / 36)$ and spa $t 441(18.4 \%, 7 / 36)$. ST59 strains were more resistant to erythromycin $(P=0.002)$ and clindamycin $(P=0.004)$ but more susceptible to ciprofloxacin $(P=0.015)$ and trimethoprim-sulfamethoxazole $(P=0.007)$ than other STs (Table 4). In addition, non-ST59 strains were more often associated with multiple antibiotic-resistance profiles. They were also resistant to moxifloxacin, rifampicin, levofloxacin, and gentamicin, while ST59 did not have these profiles.

All isolates exhibited icaA, clfA, and hla genes. The frequency of carriage for $h l b$, sea, seb, sek, seq, or sdrE among ST59 isolates was significantly higher than that for non-ST59 isolates $(P<0.05$; Table 5). Twenty-eight (77.8\%) of 36 ST59 isolates harbored $\geq 10$ tested virulence genes, which was significantly higher than that among non-ST59 isolates $(13.6 \%, 6 / 44)(P<0.05)$. However, there were no significant differences on the positive rate of $p v l$ between ST59 and non-ST59 strains.

\section{DISCUSSION}

Recently, CA-MRSA infection and transmission have become major public health problems worldwide, particularly among children and immunocompromised patients. Given the dangerous consequences of MRSA infection on pediatric patients, it is necessary to understand the prevalence, molecular characteristics and virulence profiles of these isolates in order to take effective measures to control infection and transmission in the affected communities.

Our data suggested that ST59-MRSA-IV and ST59-MRSA$\mathrm{V}$ with $t 437$ were still the most predominant clones among all of the clinical MRSA isolates in Shanghai. During recent years, CA-MRSA infections have been found worldwide and the major pandemic clones are usually related to specific geographical locations (Mediavilla et al., 2012; Li et al., 2016). For instance, the ST1 and ST8 clone in the USA and Canada, the ST30 clone in Australia, the ST80 clone in Europe, the ST59 clone in the Asia-Pacific region, including Taiwan and Australia. These five clones comprise most of the CA-MRSA worldwide. In China, previous studies of the most common genotypes revealed that ST59-MRSA-IV (and its single locus variant ST338-MRSA-IV) was the major lineage accounting for up to two-thirds of isolates and the most common spa type was $t 437$ (Geng et al., 2010). Similar to these findings, the predominant types of MRSA isolates in our study were ST59-MRSA-IV and ST59-MRSA-V with t437. The ST59 MRSA clone was also prevalent in Taiwan, Hong Kong, Vietnam, Japan, and Australia (Chuang and Huang, 2013). Based on these finding, ST59 has become a successful clone with potential for clonal expansion. However, the CA-MRSA ST59 clone identified in mainland China might differ from that identified in Taiwan (Chen and Huang, 2005). Most PVLpositive CA-MRSA ST59 clones in Taiwan belong to SCCmec $\mathrm{V}_{\mathrm{T}}$, while CA-MRSA ST59 clones in our study belong to SCCmec IV or SCCmec V. This observation supports the notion that ST59-MSSA lineages may provide a stable genetic environment for integration of SCCmec in favor of their infection and transmission in the community. In addition, the most prevalent multiresistant antibiotic profiles of ST59 strains included erythromycin, tetracycline, clindamycin, penicillin, and ampicillin. These findings may be explained by the selective pressure of antibiotic abuse in the Chinese community (Aires de Sousa et al., 2003). 
TABLE 1 | Clonal complexes and the relationship among the molecular types of MRSA isolates from pediatric patients.

\begin{tabular}{|c|c|c|c|c|c|}
\hline \multirow{2}{*}{$\begin{array}{l}\text { Clonal } \\
\text { complex (CC) }\end{array}$} & \multirow[t]{2}{*}{ MLST $(n, \%)$} & \multicolumn{4}{|c|}{ ScCmec type } \\
\hline & & spa Type & IV & $\mathbf{v}$ & NO \\
\hline \multirow[t]{9}{*}{ CC59 } & ST59 (36, 45.0\%) & $t 437$ & 14 & 3 & 17 \\
\hline & & $t 441$ & & 7 & 7 \\
\hline & & t316 & 4 & & 4 \\
\hline & & $t 172$ & 4 & & 4 \\
\hline & & $t 163$ & 1 & & 1 \\
\hline & & $t 1751$ & 1 & & 1 \\
\hline & & t3523 & 1 & & 1 \\
\hline & & $t 8886$ & 1 & & 1 \\
\hline & ST338 (2, 2.5\%) & $t 437$ & & 2 & 2 \\
\hline \multirow[t]{3}{*}{ CC398 } & ST398 (9, 11.25\%) & t034 & 2 & 4 & 6 \\
\hline & & $t 011$ & 1 & 1 & 2 \\
\hline & & $t 1250$ & & 1 & 1 \\
\hline \multirow[t]{3}{*}{ CC88 } & ST88 (7, 8.75\%) & $t 2310$ & 4 & & 4 \\
\hline & & $t 7480$ & 2 & & 2 \\
\hline & & $t 7637$ & & 1 & 1 \\
\hline \multirow[t]{5}{*}{ CC5 } & ST5 $(6,7.5 \%)$ & t062 & 3 & & 3 \\
\hline & & $t 2460$ & 1 & & 1 \\
\hline & & $t 187$ & & 1 & 1 \\
\hline & & t002 & 1 & & 1 \\
\hline & ST950 & t895 & 1 & & 1 \\
\hline \multirow[t]{2}{*}{$\mathrm{CC} 8$} & ST630 (6, 7.5\%) & $t 4549$ & 1 & 4 & 5 \\
\hline & & NT & & 1 & 1 \\
\hline \multirow[t]{2}{*}{$\mathrm{CC} 7$} & ST7 (3, 3.75\%) & t796 & & 2 & 2 \\
\hline & & t091 & 1 & & 1 \\
\hline \multirow[t]{2}{*}{ CC45 } & ST45 (2, 2.5\%) & $t 116$ & 1 & & 1 \\
\hline & & t230 & 1 & & 1 \\
\hline \multirow[t]{2}{*}{ CC72 } & ST72 & $t 664$ & 1 & & 1 \\
\hline & ST1507 & t664 & 1 & & 1 \\
\hline \multirow[t]{5}{*}{ cc9 } & ST9 (2, 2.5\%) & t899 & & 2 & 2 \\
\hline & ST30 & t019 & 1 & & 1 \\
\hline & ST6 & $t 701$ & & 1 & 1 \\
\hline & ST20 & $t 164$ & & 1 & 1 \\
\hline & ST121 & $t 159$ & & 1 & 1 \\
\hline
\end{tabular}

In this study, ST398-MRSA- IV/V with three spa types, $t 034$, $t 011$, and $t 1250$, was found to be the second most predominant type. This MRSA clone, first observed among pig and pig farmers in the Netherlands in 2003, then found in Austria, Germany, and Denmark (Fluit, 2012). Due to the presence of a restriction/methylation system, the strains are non-typeable with standard PFGE using SmaI digestion. ST398 is a typical livestockassociated type (Graveland et al., 2011), previous studies show

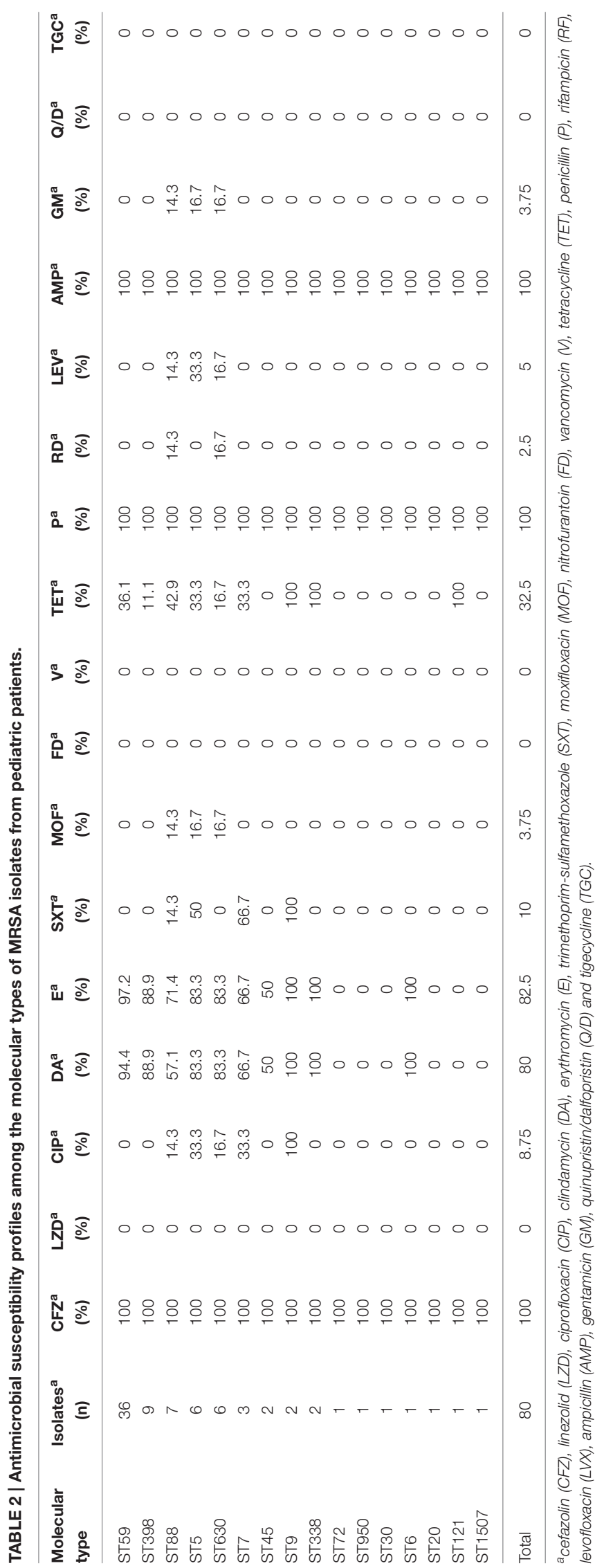




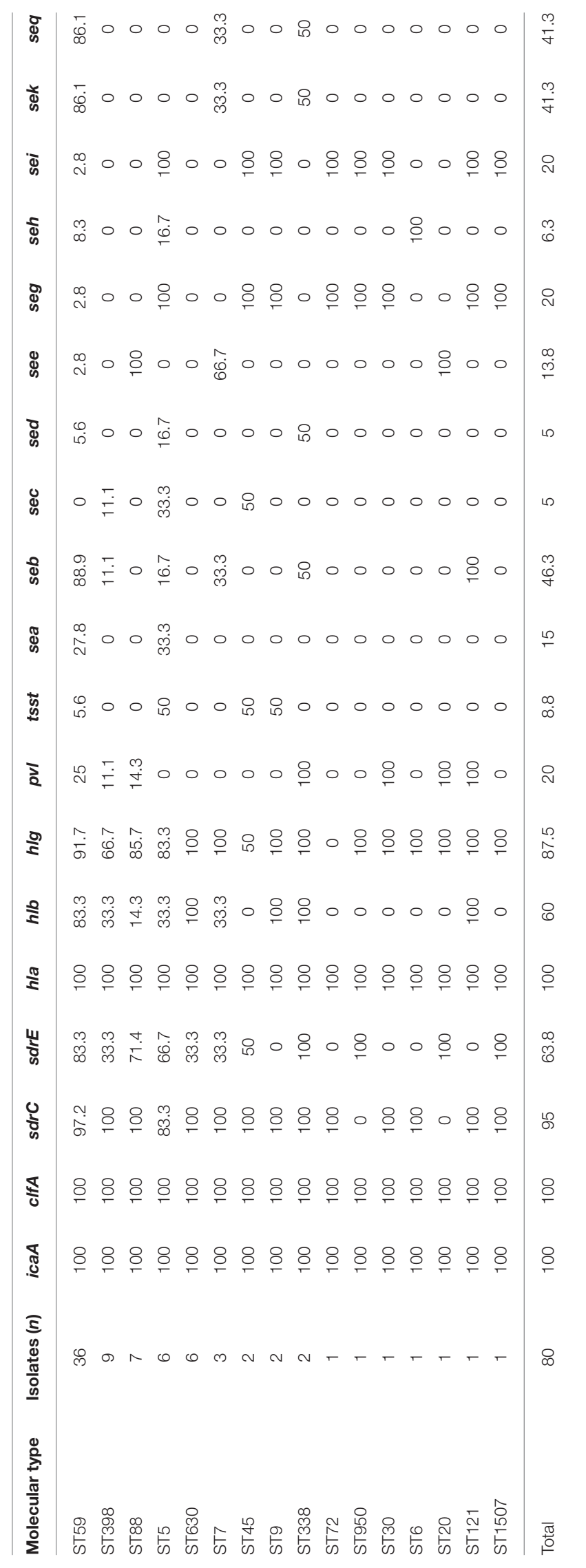

TABLE 4 | Antimicrobial susceptibility profiles of ST59 and non-ST59

isolates.

\begin{tabular}{lccc}
\hline & ST59 $(\boldsymbol{n}=\mathbf{3 6}), \mathbf{R}^{\mathbf{a}}(\mathbf{\%})$ & Non-ST59 $(\boldsymbol{n}=\mathbf{4 4}), \mathbf{R}(\%)$ & $\boldsymbol{P}^{\text {- value }} \mathbf{b}^{\mathbf{b}}$ \\
\hline CFZ & 100 & 100 & \\
LZD & 0 & 0 & \\
CIP & 0 & 15.9 & $<0.05$ \\
DA & 94.4 & 68.1 & $<0.01$ \\
E & 97.2 & 70.5 & $<0.01$ \\
SXT & 0 & 18.2 & $<0.01$ \\
MOF & 0 & 6.8 & $>0.05$ \\
FD & 0 & 0 & \\
V & 0 & 0 & \\
TET & 36.1 & 29.5 & $>0.05$ \\
P & 100 & 100 & $>0.05$ \\
RD & 0 & 4.5 & $>0.05$ \\
LEV & 0 & 9.1 & \\
AMP & 100 & 100 & $>0.05$ \\
GM & 0 & 6.8 & \\
Q/D & 0 & 0 & \\
TGC & 0 & 0 &
\end{tabular}

${ }^{a} R$, Resistance.

${ }^{b}$ The resistance rates of antimicrobials among ST59 were compared with those among non-ST59 isolates.

TABLE 5 | Frequencies of virulence genes among ST59 and non-ST59 isolates.

\begin{tabular}{lccc}
\hline Virulence genes & ST59 $(\boldsymbol{n}=\mathbf{3 6})$ & Non-ST59 $(\boldsymbol{n}=\mathbf{4 4})$ & $\boldsymbol{P}_{\text {-value }}^{\mathbf{a}}$ \\
\hline icaA & 100 & 100 & $>0.05$ \\
ClfA & 100 & 100 & $>0.05$ \\
sdrC & 97.2 & 93.2 & $>0.05$ \\
sdrE & 83.3 & 47.7 & $<0.01$ \\
hla & 100 & 100 & $>0.05$ \\
hlb & 83.3 & 40.9 & $<0.01$ \\
hlg & 91.7 & 84.1 & $>0.05$ \\
pvl & 25 & 15.9 & $>0.05$ \\
tsst & 5.6 & 11.4 & $>0.05$ \\
sea & 27.8 & 4.5 & $<0.01$ \\
seb & 88.9 & 11.4 & $<0.01$ \\
sec & 0 & 9.1 & \\
sed & 5.6 & 4.5 & $>0.05$ \\
see & 2.8 & 22.7 & $<0.01$ \\
seg & 2.8 & 34.1 & $<0.01$ \\
seh & 8.3 & 4.5 & $>0.05$ \\
sei & 2.8 & 34.1 & $<0.01$ \\
sek & 86.1 & 4.5 & $<0.01$ \\
seq & 86.1 & 4.5 & $<0.01$ \\
\hline
\end{tabular}

${ }^{a}$ The positive rates of virulence genes among ST59 were compared with those among non-ST59 isolates.

that patients carrying this type are usually in contact with a major animal reservoir of these MRSA, mostly pigs (Yan et al., 2014). In our study, six patients $(66.7 \%, 6 / 9)$ were $<6$ months and the others $(33.3 \%, 3 / 9)$ were $<3$ years. From their medical history, 
they were no directly in contact with animal hosts, suggesting that there is a potential risk of MRSA introduction from the animal reservoirs into communities or hospitals with humans as the vector. ST9 is the dominant livestock-associated lineage in Asia (Chuang and Huang, 2015), there were two ST9 strains found in our study and no evidence to support them as LAMRSA clones. In China, there is still very limited information on the occurrence of LA-MRSA infection, probably due to lack of diagnostic facilities and transmission information. Identifying risk factors and transmission routes is very important.

Based on available studies, the pathogenicity for $S$. aureus largely depends on the carriage of a battery of virulence factors associated with adhesion, invasion, persistence, the evasion/destruction of host defenses, tissue invasion/penetration and toxin-mediated disease (Moreillon et al., 1995; Bubeck Wardenburg et al., 2007; Diep and Otto, 2008; Yu et al., 2011). Of those, previous studies reported some virulence genes are found virtually in all S. aureus, while others are linked to specific molecular types. However, in our study, most of the MRSA isolates were found to harbor the hla, hlg, seb, and sek genes, but no association was found between molecular types and toxin genes. Compared to other STs, ST59 clones harbor more toxin genes and $77.8 \%$ of ST59 isolates harbored $\geq 10$ tested virulence genes, which may help ST59 become successfully spread in China. In addition, the adhesion genes ( $c l f A, i c a A, s d r C$, and $s d r E$ ) were evaluated in this study (Josefsson et al., 1998). All strains carried the $i c a A$ and $c l f A$ genes, $95 \%$ harbored $s d r C$, and $63.8 \%$ carried $s d r E$. The high percentage is consistent with the fact that these genes are ubiquitously carried by MRSA strains that belong to different lineages and that these genes have been reported to play a key role in the process of bacterial pathogenicity.

\section{REFERENCES}

Aanensen, D. M., and Spratt, B. G. (2005). The multilocus sequence typing network: mlst.net. Nucleic Acids Res. 33, W728-W733. doi: 10.1093/nar/ gki415

Aires de Sousa, M., Crisóstomo, M. I., Sanches, I. S., Wu, J. S., Fuzhong, J., Tomasz, A., et al. (2003). Frequent recovery of a single clonal type of multidrug-resistant Staphylococcus aureus from patients in two hospitals in Taiwan and China. J. Clin. Microbiol. 41, 159-163. doi: 10.1128/JCM.41.1.159-163.2003

Arvidson, S., and Tegmark, K. (2001). Regulation of virulence determinants in Staphylococcus aureus. Int. J. Med. Microbiol. 291, 159-170. doi: 10.1078/14384221-00112

Bubeck Wardenburg, J., Patel, R. J., and Schneewind, O. (2007). Surface proteins and exotoxins are required for the pathogenesis of Staphylococcus aureus pneumonia. Infect. Immun. 75, 1040-1044. doi: 10.1128/IAI. 01313-06

Chen, C. J., and Huang, Y. C. (2005). Community-acquired methicillin-resistant Staphylococcus aureus in Taiwan. J. Microbiol. Immunol. Infect. 38, 376-382.

Chen, C. J., and Huang, Y. C. (2014). New epidemiology of Staphylococcus aureus infection in Asia. Clin. Microbiol. Infect. 20, 605-623. doi: 10.1111/14690691.12705

Chuang, Y. Y., and Huang, Y. C. (2013). Molecular epidemiology of communityassociated meticillin-resistant Staphylococcus aureus in Asia. Lancet Infect. Dis. 13, 698-708. doi: 10.1016/S1473-3099(13)70136-1

Chuang, Y. Y., and Huang, Y. C. (2015). Livestock-associated meticillin-resistant Staphylococcus aureus in Asia: an emerging issue? Int. J. Antimicrob. Agents 45, 334-340. doi: 10.1016/j.ijantimicag.2014.12.007
In conclusion, simultaneous carriage of multiple virulence genes and considerable genetic diversity were very common among CA-MRSA from pediatric patients in Shanghai. ST59MRSA-IV with $t 437$ was still the most predominant type. In an era of increasing CA-MRSA, appropriate measures need to be taken to prevent further morbidity and mortality worldwide. This effort should take into account the molecular characteristics of CA-MRSA strains.

\section{AUTHOR CONTRIBUTIONS}

ML, QF, and XW designed the studies and obtained funding. XW, $\mathrm{XL}, \mathrm{WH}$, and WL performed the experiments and/or analyzed the data; ML, XW, and XL wrote the manuscript.

\section{FUNDING}

This study was supported by the National Natural Science Foundation of China (grants 81301392) and the Training Program for Outstanding Young Teachers in Higher Education Institutions (ZZjdyx13132), the Training Program for Clinical Medical Young Talents in Shanghai, Visiting Scholar Research Program and SCMC-EPT Program to XW.

\section{ACKNOWLEDGMENTS}

The authors would like to thank all the patients who contributed their specimens and clinical data for this study. We thank the microbiologists and technical staff of Shanghai Children's Medical Center for collecting the bacterial isolates and laboratory testing.

Clinical and Laboratory Standards Institute (2012). Performance Standards for Antimicrobial Suspectibility Testing. Wayne, PA: Clinical and Laboratory Standards Institute. Twenty Second Information Supplement (M100-S22).

David, M. Z., and Daum, R. S. (2010). Community-associated methicillinresistant Staphylococcus aureus: epidemiology and clinical consequences of an emerging epidemic. Clin. Microbiol. Rev. 23, 616-687. doi: 10.1128/CMR. 00081-09

DeLeo, F. R., Otto, M., Kreiswirth, B. N., and Chambers, H. F. (2010). Communityassociated meticillin-resistant Staphylococcus aureus. Lancet 375, 1557-1568. doi: 10.1016/S0140-6736(09)61999-1

Deresinski, S. (2005). Methicillin-resistant Staphylococcus aureus: an evolutionary, epidemiologic, and therapeutic odyssey. Clin. Infect. Dis. 40, 562-573. doi: $10.1086 / 427701$

Diep, B. A., and Otto, M. (2008). The role of virulence determinants in community-associated MRSA pathogenesis. Trends Microbiol. 16, 361-369. doi: 10.1016/j.tim.2008.05.002

Dinges, M. M., Orwin, P. M., and Schlievert, P. M. (2000). Exotoxins of Staphylococcus aureus. Clin. Microbiol. Rev. 13, 16-34. doi: 10.1128/CMR.13.1.16-34.2000

Enright, M. C., and Spratt, B. G. (1999). Multilocus sequence typing. Trends Microbiol. 7, 482-487. doi: 10.1016/S0966-842X(99)01609-1

Fluit, A. C. (2012). Livestock-associated Staphylococcus aureus. Clin. Microbiol. Infect. 18, 735-744. doi: 10.1111/j.1469-0691.2012.03846.x

Geng, W., Yang, Y., Wu, D., Huang, G., Wang, C., Deng, L., et al. (2010). Molecular characteristics of community-acquired, methicillin-resistant Staphylococcus aureus isolated from Chinese children. FEMS Immunol. Med. Microbiol. 58, 356-362. doi: 10.1111/j.1574-695X.2009.00648.x 
Graveland, H., Duim, B., van Duijkeren, E., Heederik, D., and Wagenaar, J. A. (2011). Livestock-associated methicillin-resistant Staphylococcus aureus in animals and humans. Int. J. Med. Microbiol. 301, 630-634. doi: 10.1016/j.ijmm.2011.09.004

Josefsson, E., McCrea, K. W., Ni Eidhin, D., O’Connell, D., Cox, J., Hook, M., et al. (1998). Three new members of the serine-aspartate repeat protein multigene family of Staphylococcus aureus. Microbiology 144(Pt 12), 3387-3395. doi: 10.1099/00221287-144-12-3387

Klevens, R. M., Morrison, M. A., Nadle, J., Petit, S., Gershman, K., Ray, S., et al. (2007). Invasive methicillin-resistant Staphylococcus aureus infections in the United States. JAMA 298, 1763-1771. doi: 10.1001/jama.298. 15.1763

Kondo, Y., Ito, T., Ma, X. X., Watanabe, S., Kreiswirth, B. N., Etienne, J., et al. (2007). Combination of multiplex PCRs for staphylococcal cassette chromosome mec type assignment: rapid identification system for mec, ccr, and major differences in junkyard regions. Antimicrob. Agents Chemother. 51, 264-274. doi: 10.1128/AAC.00165-06

Koreen, L., Ramaswamy, S. V., Graviss, E. A., Naidich, S., Musser, J. M., and Kreiswirth, B. N. (2004). spa typing method for discriminating among Staphylococcus aureus isolates: implications for use of a single marker to detect genetic micro- and macrovariation. J. Clin. Microbiol. 42, 792-799. doi: 10.1128/JCM.42.2.792-799.2004

Li, M., Dai, Y., Zhu, Y., Fu, C. L., Tan, V. Y., Wang, Y., et al. (2016). Virulence determinants associated with the Asian community-associated methicillin-resistant Staphylococcus aureus lineage ST59. Sci. Rep. 6:27899. doi: 10.1038/srep27899

Lina, G., Piémont, Y., Godail-Gamot, F., Bes, M., Peter, M. O., Gauduchon, V., et al. (1999). Involvement of Panton-Valentine leukocidin-producing Staphylococcus aureus in primary skin infections and pneumonia. Clin. Infect. Dis. 29, 1128-1132. doi: $10.1086 / 313461$

Lowy, F. D. (1998). Staphylococcus aureus infections. N. Engl. J. Med. 339, 520-532. doi: 10.1056/NEJM199808203390806

Machuca, M. A., Sosa, L. M., and Gonzalez, C. I. (2013). Molecular typing and virulence characteristic of methicillin-resistant Staphylococcus aureus isolates from pediatric patients in Bucaramanga, Colombia. PLoS ONE 8:e73434. doi: 10.1371/journal.pone.0073434

Mediavilla, J. R., Chen, L., Mathema, B., and Kreiswirth, B. N. (2012). Global epidemiology of community-associated methicillin resistant
Staphylococcus aureus (CA-MRSA). Curr. Opin. Microbiol. 15, 588-595. doi: 10.1016/j.mib.2012.08.003

Monecke, S., Coombs, G., Shore, A. C., Coleman, D. C., Akpaka, P., Borg, M., et al. (2011). A field guide to pandemic, epidemic and sporadic clones of methicillin-resistant Staphylococcus aureus. PLoS ONE 6:e17936. doi: 10.1371/journal.pone.0017936

Moreillon, P., Entenza, J. M., Francioli, P., McDevitt, D., Foster, T. J., Francois, P., et al. (1995). Role of Staphylococcus aureus coagulase and clumping factor in pathogenesis of experimental endocarditis. Infect. Immun. 63, 4738-4743.

Peacock, S. J., Moore, C. E., Justice, A., Kantzanou, M., Story, L., Mackie, K. et al. (2002). Virulent combinations of adhesin and toxin genes in natural populations of Staphylococcus aureus. Infect. Immun. 70, 4987-4996. doi: 10.1128/IAI.70.9.4987-4996.2002

Shopsin, B., Gomez, M., Montgomery, S. O., Smith, D. H., Waddington, M., Dodge, D. E., et al. (1999). Evaluation of protein A gene polymorphic region DNA sequencing for typing of Staphylococcus aureus strains. J. Clin. Microbiol. 37, 3556-3563.

Witte, W. (2009). Community-acquired methicillin-resistant Staphylococcus aureus: what do we need to know? Clin. Microbiol. Infect. 15(Suppl. 7), 17-25. doi: 10.1111/j.1469-0691.2009.03097.x

Yan, X., Yu, X., Tao, X., Zhang, J., Zhang, B., Dong, R., et al. (2014). Staphylococcus aureus ST398 from slaughter pigs in northeast China. Int. J. Med. Microbiol. 304, 379-383. doi: 10.1016/j.ijmm.2013.12.003

Yu, F., Yang, L., Pan, J., Chen, C., Du, J., Li, Q., et al. (2011). Prevalence of virulence genes among invasive and colonising Staphylococcus aureus isolates. J. Hosp. Infect. 77, 89-91. doi: 10.1016/j.jhin.2010.07.019

Conflict of Interest Statement: The authors declare that the research was conducted in the absence of any commercial or financial relationships that could be construed as a potential conflict of interest.

Copyright (c) 2016 Wang, Li, Liu, Huang, Fu and Li. This is an open-access article distributed under the terms of the Creative Commons Attribution License (CC BY). The use, distribution or reproduction in other forums is permitted, provided the original author(s) or licensor are credited and that the original publication in this journal is cited, in accordance with accepted academic practice. No use, distribution or reproduction is permitted which does not comply with these terms. 